\title{
Habilidades básicas e desempenho acadêmico em universitários ingressantes
}

\author{
Ricardo Primi \\ Acácia A. Angeli dos Santos. \\ Claudette Medeiros Vendramini
}

Universidade São Francisco

\begin{abstract}
Resumo
Recentes estudos sobre o desenvolvimento cognitivo adulto referem-se à distinção entre inteligência fluida como a capacidade geral de relacionar idéias complexas, formar conceitos abstratos e derivar implicações lógicas a partir de regras gerais e inteligência cristalizada como a capacidade de derivar conhecimento a partir de esquemas organizados de informações sobre disciplinas específicas. Para verificar a possível relação entre a habilidade cognitiva requerida e a área de conhecimento, este estudo foi proposto com o objetivo de investigar as correlações entre medidas de inteligência fluida e cristalizada com desempenho acadêmico em 960 alunos ingressantes dos cursos de Medicina, Odontologia, Engenharia Civil, Matemática, Psicologia, Pedagogia, Letras e Administração. As correlações encontradas indicam que o desempenho acadêmico está associado a diferentes perfis de habilidades cognitivas.
\end{abstract}

Palavras-chave: Habilidades básicas, Inteligência fluida e cristalizada, Avaliação educacional, Teorias de inteligência.

\begin{abstract}
Basic abilities and scholastic achievement in freshmen students

Recent studies of adult cognitive development have distinguished two core abilities: fluid intelligence as a general capacity to infer complex relationships, abstract concepts, and to deduce logical implications from general rules; and crystallized intelligence as a capacity to solve problems by using organized knowledge schemes from specific disciplines. In order to investigate a possible relationship between cognitive abilities and area of study, this research studied the correlation between fluid and crystallized intelligence measures and academic achievement among 960 freshmen students of eight areas of study: Medicine, Dentistry, Psychology, Business, Engineering, Mathematics, Education, and Literature. The results have indicated that the academic achievement is correlated with different abilities profile.
\end{abstract}

Key words: Basic abilities, Fluid and crystallized intelligence, Educational assessment, Intelligence theories.

$\mathrm{C}$ om as transformações pelas quais a sociedade tem passado, cada vez mais tem sido apontado que será necessário ao profissional das próximas gerações, não apenas o domínio de conhecimentos específicos, mas a capacidade de se adaptar rapidamente e assimilar novas informações de um mundo em constante transformação. Isso vem se tornando um pré-requisito básico para o perfil do profissional do novo milênio. Portanto, a universidade deverá, cada vez mais, produzir estratégias que privilegiem, não só a aprendizagem de conteúdos, mas também a aprendizagem de estratégias de adaptação a situações novas.

Essa capacidade, definida pela psicologia como "inteligência geral", foi alvo de várias pesquisas desde o início deste século (Almeida, 1988; Sternberg, 1981). Os estudos psicométricos, que investigaram como as habilidades humanas se estruturam, chamaram-na de fator g, enquanto em estudos mais recentes desenvolvidos pela psicologia cognitiva, vários dos processos que poderiam ser classifica- 
dos como gerais voltam a ser revistos e entendidos como formas de adaptação a situações novas.

Tomando como objeto de estudo os testes de inteligência desenvolvidos pelos psicometristas, Sternberg (1986) divide-os em dois grandes grupos: os testes que utilizam tarefas de raciocínio indutivo e os testes que utilizam tarefas de raciocínio dedutivo. Dentro do primeiro grupo são incluídas tarefas de classificação (formação de conceitos), seriação, entendimento de metáforas e analogias; e, no segundo grupo, as tarefas associadas à lógica categórica (silogismos), hipotéticas e disjuntivas (Copi, 1968).

Retomando-se a história das teorias da inteligência, pode-se observar enfoques diferenciados em capacidades mais associadas ao raciocínio ou ao conhecimento. Essas dimensões da inteligência humana têm sido referidas, desde Cattell (1971), por inteligência fluida (raciocínio) e cristalizada (conhecimento). A primeira refere-se à capacidade de processamento cognitivo, isto é, à capacidade geral de relacionar idéias complexas, formar conceitos abstratos e derivar implicações lógicas a partir de regras gerais em situações relativamente novas (para as quais existem poucos conhecimentos previamente memorizados). A segunda, refere-se à extensão e à profundidade das informações adquiridas, via escolarização que, geralmente, são usadas na resolução de problemas semelhantes aos que se aprendeu no passado, ou ao "estoque" acumulado de conhecimentos, isto é, esquemas organizados de informações sobre áreas específicas do conhecimento (Ackerman, 1996; Ackerman \& Heggestad, 1997; Ackerman, Kyllonen \& Roberts, 1999; Carrol, 1993; Horn, 1991; McGrew \& Flanagan, 1998).

Ackerman (1996) analisa que a testagem da inteligência no início do século pautou-se na avaliação dos processos cognitivos, observando o raciocínio que as pessoas empenhavam quando resolviam problemas, diferentemente de métodos anteriores, voltados mais à avaliação do conhecimento, isto é, o que as pessoas sabiam. Tal orientação culminou na abordagem do processamento da informação na década de setenta (Primi, 1995a, 1995b, 1998; Primi \& Rosado, 1995; Sternberg, 1977).

Alguns autores criticam esta visão processual, argumentando que ela deu pouca importância ao conhecimento e que o processamento nunca ocorre em um vácuo de conteúdo, pois sempre pressupõe um tema ou conteúdo específico sobre o qual se raciocina. Assim, a avaliação da inteligência nunca pode prescindir do conhecimento, porque sempre se é inteligente em alguma área do conhecimento. Os integrantes desta corrente consideram que as medidas de conhecimento são tão preditivas quanto aquelas medidas mais "purificadas" do raciocínio, geralmente testes de inteligência fluida (Chen \& Gardner, 1997; Gardner, 1995; Rolfhus \& Ackerman, 1996, 1999).
Por outro lado, com as transformações sociais, o conhecimento passa a ser muito mais volátil e dinâmico, exigindo cada vez mais a capacidade de raciocínio para que novas informações possam ser rapidamente aprendidas. Nesse cenário, o conhecimento prévio não é o determinante da capacidade de adaptação e aprendizagem.

Como sabemos, grande parte dos processos seletivos no Brasil têm privilegiado a noção de inteligência como capacidade de conhecimento (inteligência cristalizada). Nos Estados Unidos ocorre o contrário: o Scholastic Assessment Test (SAT I) enfatiza mais a capacidade de raciocínio utilizando sub-testes de raciocínio verbal (analogias, completar sentenças e leitura crítica) e raciocínio matemático (problemas de aritmética, álgebra, geometria e raciocínio lógico). Um dos propósitos deste instrumento, construído na década de quarenta, foi a avaliação de aptidões para o desempenho escolar e não a avaliação de realização escolar (Wainer, 1990). Recentemente, esta característica do SAT, ou sua maior proximidade aos testes de inteligência do que aos testes de conhecimento, tem sido alvo de críticas por aqueles que defendem avaliações centradas em conteúdos tratados no currículo, como resume Cloud (2001). No Brasil, um novo sistema de avaliação, o Exame Nacional do Ensino Médio (ENEM), parece ter mudado esta concepção, situando-se em algum lugar entre o propósito de avaliar o raciocínio e o conhecimento (Primi et al., no prelo).

Pensando no desempenho do aluno no contexto universitário, pode-se questionar: qual a importância relativa de medidas mais associadas ao conhecimento, como a das provas tradicionais, e de outras, associadas ao raciocínio? Reconhecendo-se a importância destas duas capacidades, duas hipóteses podem ser levantadas: a primeira, enfatizando o conhecimento, pressupõe que quanto mais profundo e extenso for o conhecimento do aluno sobre o conteúdo tratado no ensino médio, mais preparado ele estará para prosseguir no ensino universitário e, portanto, maior será o seu desempenho. A segunda, enfatizando o raciocínio, parte do princípio de que, como o conteúdo tratado na universidade será novo, quanto maior a capacidade de raciocínio do aluno, mais bem preparado ele estará para organizar as novas informações e, portanto, maior será seu desempenho.

Pode-se indagar, também, se a importância relativa destas habilidades é a mesma se considerarmos o desempenho acadêmico em diferentes cursos universitários. Considerando que o desempenho em cursos distintos associa-se ao domínio de conteúdos também distintos, pode-se supor que a importância relativa das duas habilidades mencionadas pode mudar se analisarmos o desempenho separadamente em diferentes cursos.

O presente estudo objetivou explorar as correlações existentes entre medidas associadas à inteligência cristali- 
zada e medidas associadas à inteligência fluida, buscando investigar a importância relativa dessas medidas na previsão do desempenho acadêmico em diferentes cursos universitários.

\section{Método}

\section{Participantes}

Analisou-se o desempenho de 960 alunos ingressantes dos cursos de Medicina, Odontologia, Engenharia Civil, Matemática, Psicologia, Pedagogia, Letras e Administração, com idades variando de 17 a 50 anos (média 21,9 e desvio padrão 4,9), dos quais $73,5 \%$ tinham de 17 a 22 anos e $65,9 \%$ eram do sexo feminino.

\section{Instrumentos e procedimentos}

Como parte do processo seletivo, os alunos responderam a provas de conhecimento em dez disciplinas. Essas provas consistiam de 310 questões abrangendo os seguintes conteúdos: Língua Portuguesa (40), Literatura Brasileira (30), Biologia (30), Física (30), Língua Inglesa (30), Matemática (30), Química (30), História (30), Geografia (30), Conhecimentos Gerais (30). Os alunos deviam escolher e responder 210 questões dessas 310 que eram oferecidas. Após uma análise fatorial exploratória, as notas nessas provas foram organizadas em três escores fatoriais: Linguagem Verbal - LV (incluindo as provas de Português, Literatura, Inglês); Conhecimento Natural



Figura 1. Exemplos dos Itens das Provas RI, CL e RLD 
Matemático - CNM (incluindo as provas de Matemática, Física, Química e Biologia) e Conhecimentos Sócio-Geográficos - CSG (incluindo as provas de Conhecimentos Gerais, História e Geografia). Também se calculou a pontuação geral que foi denominada Prosel (nota geral no processo seletivo).

No primeiro semestre, foram aplicadas três provas enfatizando a capacidade de raciocínio: (1) raciocínio indutivo (RI) avaliando a capacidade de analisar os elementos de um problema para descobrir as regularidades, inferindo as regras gerais; (2) raciocínio lógico dedutivo (RLD) avaliando a capacidade de trabalhar com as regras de um problema, combinando-as para a produção de conclusões lógicas; e (3) compreensão em leitura (CL) avaliando a habilidade geral de ler e compreender as idéias presentes no texto. Exemplos de itens dessas provas são apresentados na Figura 1.

Como medida do desempenho acadêmico, foi calculado para cada aluno o desempenho médio nas disciplinas cursadas nos dois primeiros semestres de seu curso. Essa variável foi chamada rendimento médio geral (RMG).

As provas de raciocínio foram aplicadas como parte de um projeto mais amplo de avaliação de habilidades básicas de universitários, do qual os alunos participaram voluntariamente. Fez parte deste projeto uma devolutiva individual do desempenho nessas provas por meio de carta. $\mathrm{Na}$ análise dos dados, focalizou-se o desempenho acadêmico, calculando-se as correlações entre as medidas do processo seletivo e das provas de raciocínio com o desempenho acadêmico.

Tabela 1. Estatísticas descritivas das medidas de inteligência cristalizada e fluida

\begin{tabular}{lrrrrrrrr}
\hline & CL & RI & RLD & CNM & LV & CSG & Prosel & RMG \\
\hline Medicina (N=72) & & & & & & & & \\
M & 569 & 612 & 630 & 757 & 565 & 578 & 752 & 7,19 \\
DP & 650 & 77 & 55 & 91 & 132 & 102 & 60 & 0,54 \\
Odontologia (N=154) & & & & & & & & \\
M & 466 & 476 & 546 & 536 & 511 & 539 & 548 & 5,62 \\
DP & 93 & 86 & 68 & 78 & 106 & 94 & 59 & 0,68 \\
Administração (N=362) & & & & & & & & \\
M & 487 & 494 & 468 & 473 & 478 & 475 & 458 & 6,09 \\
DP & 100 & 98 & 94 & 52 & 83 & 89 & 5691 & 1,25 \\
Pedagogia (N=67) & & & & & & & & \\
M & 524 & 442 & 486 & 456 & 481 & 481 & 450 & 7,40 \\
DP & 84 & 96 & 89 & 59 & 79 & 112 & 76 & 0,62 \\
Psicologia (N=176) & & & & & & & & \\
M & 524 & 529 & 503 & 454 & 522 & 516 & 486 & 6,91 \\
DP & 86 & 88 & 92 & 58 & 103 & 102 & 70 & 0,70 \\
Letras (N=51) & & & & & & & & \\
M & 541 & 510 & 531 & 457 & 538 & 475 & 473 & 6,80 \\
DP & 116 & 84 & 86 & 65 & 111 & 82 & 71 & 1,02 \\
Eng. Civil (N=46) & & & & & & & & \\
M & 457 & 531 & 494 & 495 & 471 & 477 & 474 & 5,83 \\
DP & 120 & 101 & 94 & 62 & 86 & 84 & 55 & 1,19 \\
Matemática (N=32) & & & & & & & & \\
M & 504 & 472 & & 481 & 457 & 445 & 439 & 5,89 \\
DP & & 79 & & 48 & 66 & 87 & 51 & 1,20 \\
\hline
\end{tabular}

Nota: os dados da prova RLD para o curso de matemática não puderam ser coletados em função de limitações no calendário do próprio curso. 


\section{Resultados}

Na Tabela 1, apresentam-se as médias e desvios padrão nas variáveis analisadas, dividindo-se os 960 alunos conforme o curso. Com exceção do rendimento médio geral (RMG), as notas nas provas foram padronizadas para a escala CEEB (College Entrance Examination Board, Anastasi \& Urbina, 1997), na qual as notas variam de 200 a 800 com média 500 e desvio padrão 100 . As notas no RMG foram apresentadas em sua escala habitual de 0 a 10 .

Como pode ser observado, nos cursos com alto grau de competitividade (com relação candidato-vaga alta, caso da Medicina), os alunos tenderam a apresentar desempenho acima da média (valores maiores que 500), sobretudo nas provas do processo seletivo. Algumas diferenças entre as habilidades nos diversos cursos são notáveis: os alunos do curso de Odontologia tenderam a apresentar notas acima da média nas provas relacionadas à linguagem (RLD e LV) e conhecimentos naturais matemáticos (CNM) e sócio-geográficos (CSG). De modo semelhante, os alunos de Letras tenderam a apresentar notas acima da média em habilidades relacionadas à linguagem, tais como, CL, RLD e LV. Os alunos da Psicologia tenderam a apresentar notas acima da média nas provas RI e CL e os alunos da Engenharia Civil, na prova RI.

Na Tabela 2, apresentam-se as correlações entre as provas aplicadas e o desempenho médio geral. Como pode ser observado, vários coeficientes foram significativamente diferentes de zero, evidenciando que as medidas coletadas contribuem para a previsão do desempenho

Tabela 2. Coeficientes de correlação entre as provas de raciocínio e conhecimento com o rendimento médio geral

\begin{tabular}{|c|c|c|c|c|c|c|c|}
\hline & $\mathrm{CL}$ & RI & RLD & $\mathrm{CNM}$ & LV & CSG & Prosel \\
\hline \multicolumn{8}{|c|}{ Medicina } \\
\hline$r$ & 0,007 & 0,205 & 0,215 & 0,095 & $-0,067$ & 0,033 & $-0,024$ \\
\hline$N$ & 54 & 55 & 68 & 72 & 72 & 72 & 72 \\
\hline \multicolumn{8}{|c|}{ Odontologia } \\
\hline$r$ & $* * * 0,286$ & $* 0,173$ & 0,193 & $* 0,195$ & 0,137 & 0,009 & $* * * 0,279$ \\
\hline$N$ & 137 & 145 & 82 & 154 & 154 & 154 & 154 \\
\hline \multicolumn{8}{|c|}{ Administração } \\
\hline$r$ & $* * * 0,220$ & $* * * 0,254$ & $* * * 0,266$ & 0,058 & $* * 0,159$ & 0,091 & $* * * 0,238$ \\
\hline$N$ & 261 & 265 & 327 & 356 & 356 & 356 & 356 \\
\hline \multicolumn{8}{|c|}{ Pedagogia } \\
\hline$r$ & 0,069 & $-0,032$ & 0,222 & $-0,168$ & 0,183 & $* 0,294$ & $* 0,243$ \\
\hline$N$ & 54 & 53 & 64 & 67 & 67 & 67 & 67 \\
\hline \multicolumn{8}{|c|}{ Psicologia } \\
\hline$r$ & $* * 0,256$ & $* * * 0,284$ & $* * * 0,427$ & 0,019 & $* * * 0,276$ & 0,098 & $* * * 0,287$ \\
\hline$N$ & 164 & 166 & 110 & 176 & 176 & 176 & 176 \\
\hline \multicolumn{8}{|c|}{ Letras } \\
\hline$r$ & $* * 0,442$ & $* * 0,445$ & $* * * 0,503$ & $-0,236$ & $* * * 0,633$ & $* * * 0,480$ & $* * * 0,634$ \\
\hline$N$ & 43 & 43 & 48 & 51 & 51 & 51 & 51 \\
\hline \multicolumn{8}{|c|}{ Eng. Civil } \\
\hline$r$ & 0,200 & $* * 0,410$ & 0,276 & $* 0,361$ & 0,061 & $-0,096$ & 0,259 \\
\hline$N$ & 41 & 44 & 39 & 46 & 46 & 46 & 46 \\
\hline \multicolumn{8}{|c|}{ Matemática } \\
\hline$r$ & 0,294 & $* * * 0,588$ & & 0,145 & 0,037 & 0,035 & 0,190 \\
\hline$N$ & 30 & 30 & & 31 & 31 & 31 & 31 \\
\hline
\end{tabular}

$* p<0,05 ; * * p<0,01 ; * * * p<0,001$. 
geral no primeiro semestre. No entanto, dependendo do curso considerado, diferentes habilidades se salientam, indicando que o desempenho depende de habilidades diferentes relativas ao curso considerado. Por exemplo, no curso de Medicina, nenhum coeficiente foi significativo, provavelmente pela restrição da variabilidade. Entretanto, as associações de maior magnitude ocorreram com as provas $\operatorname{RLD}(p=0,08)$ e $\mathrm{RI}(p=0,13)$. No curso de Letras, as provas que contribuíram com maior magnitude foram LV e RLD. Já nos cursos de Engenharia Civil e de Matemática, a que contribui com maior magnitude foi a RI.

A Tabela 2 indica quais habilidades são mais importantes na previsão do desempenho acadêmico. Cada linha, correspondente a um determinado curso, contém as associações das sete medidas efetuadas (CL, RI, RLD, CNM, LV, CSG e Prosel) com o desempenho acadêmico. Esta linha pode ser interpretada como um vetor em um espaço de sete dimensões. Cada dimensão é vinculada a uma habilidade variando de -1 a +1 . Cada curso pode ser compreendido como um ponto nesse espaço multidimensional, cuja localização, determinada pelo vetor, dependerá do perfil de habilidades mais e menos associadas ao desempenho. Cursos, cujos pontos representados nesse espaço estejam próximos, serão semelhantes nas habilidades que mais se associam ao bom aproveitamento nas atividades acadêmicas desenvolvidas.
Por meio da análise multidimensional não-métrica foi possível reduzir o espaço com sete dimensões a um espaço bidimensional. Apresentado na Figura 2, ele reproduziu com muita eficácia as semelhanças e diferenças entre os cursos (Stress $=0,033$ e RSQ $=0,993)^{1}$.

Antes da análise, os vetores para os cursos com os coeficientes de correlação foram padronizados para que a magnitude dos coeficientes não contribuísse para diferenciá-los. Portanto, a configuração da Figura 2 representa diferenças entre a importância relativa das seis habilidades avaliadas para cada curso (a nota do Processo Seletivo foi desconsiderada nesta análise, pois representa a soma das notas CNM, LV e CSG consistindo, portanto, em informação redundante).

A análise desse gráfico sugere três agrupamentos, nos quais a importância relativa de determinadas habilidades é semelhante: o primeiro, formado pelos cursos Medicina, Matemática e Engenharia Civil, nos quais as provas RI, RLD e CNM estão relativamente mais associadas ao desempenho. O segundo, formado pelos cursos de Administração e Psicologia, nos quais as provas RLD, RI, CL e LV estão relativamente mais associadas ao desempenho. E o terceiro, formado pelos cursos de Letras e Pedagogia, nos quais as provas LV, RLD e CSG estão relativamente mais associadas ao desempenho. $\mathrm{O}$ curso de Odontologia, no qual as provas mais associadas ao

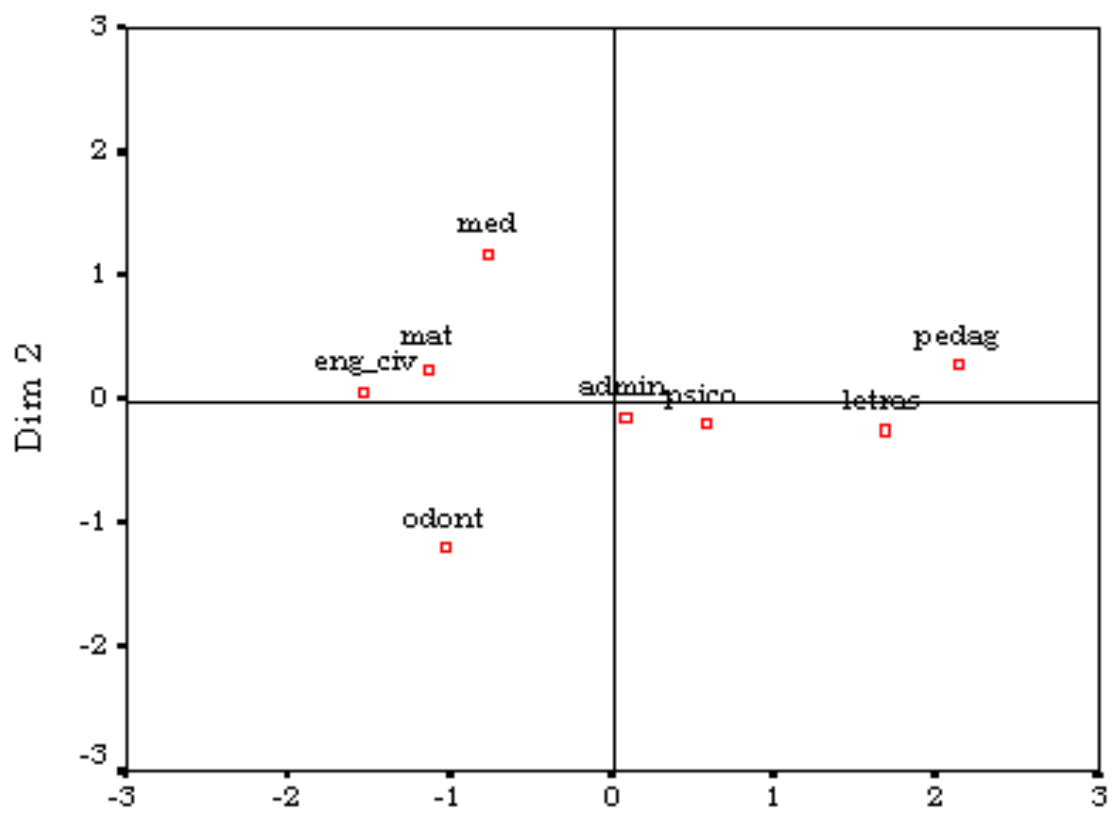

\section{$\operatorname{Dim} 1$}

Figura 2. Espaço resultante da análise multidimensional representando as semelhanças e diferenças entre os cursos nas habilidades associadas ao bom desempenho acadêmico. 
desempenho são CL, CNM e RI, está mais próximo do primeiro agrupamento por causa das provas CNM e RI, mas se distingue dele por causa da importância da prova CL.

Uma relação interessante pode ser observada nesse gráfico. A reta horizontal contrasta, à esquerda, cursos nos quais o aproveitamento acadêmico depende mais do raciocínio (inteligência fluida), com os cursos à direita, nos quais o aproveitamento está mais associado a habilidades verbais (inteligência cristalizada). No ponto intermediário estão os cursos nos quais o desempenho requer simultaneamente raciocínio e linguagem como mostra a maior importância da prova RLD.

\section{Discussão e Conclusão}

O presente estudo explorou correlações existentes entre medidas de inteligência fluida e cristalizada com o desempenho acadêmico, buscando investigar a importância relativa destas medidas na sua previsão. Os resultados sugerem que, dependendo do curso considerado, diferentes configurações de habilidades emergem como mais importantes.

Em alguns cursos (Medicina, Engenharia Civil e Matemática), o aproveitamento acadêmico está mais fortemente associado à inteligência fluida. Vale lembrar que para MacGrew e Flanagan (1998), a inteligência fluida refere-se às operações mentais que uma pessoa utiliza quando enfrenta situações relativamente novas, nas quais os conhecimentos habituais não são suficientes. Relaciona-se à formação de conceitos novos, identificação de relações, percepção de relações em padrões, estabelecimento de inferências, compreensão de implicações, resolução de problemas, extrapolação, reorganização e transformação das informações. Os resultados também indicaram uma importância significativa, mas de menor magnitude, de conhecimentos associados à matemática, física, química e biologia avaliados no processo seletivo.

De outro lado, o aproveitamento de cursos como Letras e Pedagogia está mais associado à inteligência cristalizada. Para MacGrew e Flanagan (1998), a inteligência cristalizada refere-se à extensão e à profundidade do conhecimento cultural adquirido e à capacidade de sua efetiva aplicação, referindo-se a um estoque organizado de conhecimento declarativo (informações, fatos, conceitos, regras) e, também, ao conhecimento de procedimentos de ação para situações conhecidas (situações habituais). Um fato importante é que este conhecimento é mediado principalmente pela linguagem verbal. Nestes cursos, o aproveitamento está relacionado mais fortemente com os conhecimentos avaliados pelas provas de Português, Literatura e Inglês (para o curso de Letras) e His- tória, Geografia e Conhecimentos Gerais (para o curso de Pedagogia) aplicadas no Processo Seletivo.

O aproveitamento acadêmico nos cursos de Administração e Psicologia parece depender simultaneamente das inteligências fluida e cristalizada. Por isso, situa-se em um ponto intermediário na dimensão horizontal da Figura 2. Uma evidência a favor desta interpretação é a importância da prova RLD. Esta prova avalia o fator Raciocínio Geral Seqüencial, do Estrato I do modelo de Carrol (1993), referindo-se à habilidade para trabalhar com regras ou proposições verbais, combinando-as seqüencialmente em um ou mais passos para a produção de conclusões lógicas derivadas das relações entre as premissas. Os estudos psicométricos mostram que este tipo de prova se associa simultaneamente à inteligência fluida, por causa do relacionamento de idéias, e à inteligência cristalizada por causa do envolvimento da linguagem verbal (Santos et al. 2000; Horn, 1991). Uma segunda evidência é a importância das provas RI, RLD, associadas à inteligência fluida e a das provas CL e LV, associadas à inteligência cristalizada para a previsão do aproveitamento acadêmico.

O curso de Odontologia diferiu dos outros por causa de uma combinação específica de habilidades mais associadas ao aproveitamento acadêmico. Ele se assemelha ao grupo formado pelos cursos de Medicina, Matemática e Engenharia Civil por causa da contribuição de conhecimentos de Biologia, Matemática, Física e Química, mas difere deste grupo porque a prova RI, mais associada à inteligência fluida, não se mostrou tão importante na previsão do desempenho. As habilidades mais importantes para o desempenho neste curso foram aquelas avaliadas pelas provas CL (compreensão em leitura) e RLD (raciocínio lógico-dedutivo). Estes resultados indicam uma importância maior da inteligência cristalizada, já que a prova $\mathrm{CL}$, conforme observação em estudos anteriores, está associada à extensão e à profundidade do conhecimento semântico lexical verbal dos alunos e à capacidade de recuperação deste conhecimento, disponível na memória de longo prazo (Santos, Primi, Taxa \& Vendramini, 2001).

Os dados encontrados neste estudo estão de acordo com os achados de Ackerman, (1996); Ackerman e Heggestad (1997), Ackerman, Kyllonen e Roberts (1999) sobre a progressiva diferenciação do intelecto no desenvolvimento cognitivo adulto, cujos estudos têm investigado as relações entre habilidade, interesses e personalidade. Mostram que interesses realistas (preferindo realizações observáveis e concretas, trabalhos com máquinas, eventos e coisas) e investigativos (voltado à exploração intelectual e preferindo mais o pensar do que o agir) estão associados à inteligência fluida. Interesses 
artísticos (interesse por atividades artísticas, musicais e literárias; atividades expressivas individuais, criatividade, estética e emoções) estão mais associados a habilidades verbais (inteligência cristalizada). No presente estudo, esta distinção associou-se respectivamente aos dois grupos extremos formados pelos cursos Medicina, Engenharia e Matemática, de um lado, e Pedagogia e Letras, de outro.

Retomando a questão levantada sobre o tipo de inteligência que seria relativamente mais importante na previsão do desempenho acadêmico, pode-se concluir que dependendo do curso para o qual se deseja prever o desempenho, uma delas sobressai como mais importante, sugerindo uma diferenciação das habilidades cognitivas à medida que o aluno progride no seu percurso acadêmico.

\section{Referências}

Ackerman, P. L. (1996). A theory of intellectual development: process, personality, interests, and knowledge. Intelligence, 22, 227-257.

Ackerman, P. L., \& Heggestad, E. D. (1997). Intelligence, personality, and interests: Evidence for overlapping traits. Psychological Bulletin, 121, 219-245.

Ackerman, P. L., Kyllonen, P. C., \& Roberts, R. D. (1999). Learning and individual differences. Washington: American Psychological Association.

Almeida, L. S. (1988). Teorias da Inteligência. Porto: Edições Jornal de Psicologia.

Anastasi, A., \& Urbina, S. (1997). Psychological Testing. Upper Saddle River: Prentice Hall.

Carrol, J. B. (1993). Human cognitive abilities: A survey of factor-analytic studies. New York: Cambridge University Press.

Cloud, J. (2001, 10 de março). Should SATs Matter? Time, 157 (10), [Revista on-line] Retirado em 15/05/2001 no World Wide Web: http:/ /www.time.com/time/education/article/0,8599,101321-1,00.html

Cattell, R. B. (1971). Abilities: Their structure, growth and action. Boston: Houghton Mifflin.

Chen, J. Q., \& Gardner, H. (1997). Alternative assessment from a multiple intelligences theoretical perspective. In D. P. Flanagan, J. L. Genshaft \& P. L. Harrison (Orgs.), Contemporary intellectual assessment: theories, tests, and issues (pp. 105-121). New York: The Guilford Press.

Copi, I. M. (1968). Introdução à Lógica. São Paulo: Mestre Jou.

Gardner, H. (1995). Inteligências múltiplas: a teoria na prática. Porto Alegre: Artes Médicas
Horn, J. H. (1991). Measurement of intellectual capabilities: A review of theory. In K. S. McGrew, J. K. Werder \& R. W. Woodcock (Orgs.), WJ-R Technical Manual (pp. 197-245). Allen: DLM.

Kruskal, J. B. (1964). Multidimensional scaling by optimizing goodness of fit to a nonmetric hypothesis. Psychometrika, 29, 1-27.

McGrew, K. S., \& Flanagan, D. P. (1998). The intelligence test desk reference (ITDR): Gf-Gc cross-battery assessment. Needham Heihts: Allyn \& Bacon.

Primi, R. (1995a). Inteligência, processamento de informação e teoria da gestalt: um estudo experimental. Dissertação de mestrado nãopublicada. Pontifícia Universidade Católica de Campinas, Campinas.

Primi, R. (1995b). A teoria da Gestalt e as visões componenciais da Inteligência. In R. S. L. Guzzo, G. P.Witter, S. Pfromm Netto, E. Rosado \& S. Wechsler (Orgs.), O futuro da criança na escola, família e sociedade (pp. 287-290). Campinas: Átomo/Associação Brasileira de Psicologia Escolar/International School Psychology Association.

Primi, R. (1998). Avanços tecnológicos na avaliação da inteligência. In F. C. Capovilla, M. J. Gonçalves \& E. C. Macedo (Orgs.), Tecnologia em (re) habilitação cognitiva (pp 159-166). São Paulo: EDUNISC.

Primi, R., \& Rosado, E. M. S. (1995). Os princípios de organização perceptual e a atividade inteligente: um estudo sobre testes de inteligência. Estudos de Psicologia, 11(2), 3-12.

Primi, R.; Santos , A. A. A., Vendramini, C. M., Taxa, F., Muller, F., Lukjanenko, M. F., \& Sampaio, I. (no prelo). Competências e habilidades cognitivas: diferentes definições dos mesmos construtos. Psicologia: Teoria e Pesquisa.

Rolfhus, E. L., \& Ackerman, P. L. (1996). Self-report knowledge: At the crossroads of ability, interest, and personality. Journal of Educational Psychology, 88, 174-188.

Rolfhus, E. L., \& Ackerman, P. L. (1999). Assessing individual differences in knowledge: Knowledge, intelligence, and related traits. Journal of Educational Psychology, 91, 511-526.

Santos, A. A. A., Primi, R., Vendramini, C. M., Taxa, F., Lukjanenko, M. F., Muller, F., Sampaio, I., Andraus Jr., S., Kuse, F. K., \& Bueno, C. H. (2000). Habilidades básicas de ingressantes universitários. Avaliação: Revista da Rede de Avaliação Institucional da Educação Superior, 2(16), 33-45.

Santos, A. A. A., Primi, R., Taxa, F. O. S., \& Vendramini, C. M. M. (2001). O Teste de Cloze na avaliação da compreensão em leitura. Manuscrito submetido para publicação.

Sternberg, R. J. (1981). The evolution of theories of intelligence. Intelligence, $5,209-230$.

Sternberg, R. J. (1977). A component process in analogical reasoning. Psychological Review, 84(4), 353-378.

Sternberg, R. J. (1986). Toward an unified theory of human reasoning. Intelligence, 10, 281-314.

Wainer, H. (Org.) (1990). Computerized adaptive testing: A primer. Hillsdale: Lawrence Erlbaum Associates. configuração bidimensional final representa adequadamente as distâncias originais entre os cursos originadas do espaço com sete dimensões. O stress é o resíduo resultante da comparação das distâncias reproduzidas com as observadas sendo esperado valores pequenos, caso a configuração final represente adequadamente as distâncias originais. O RQS é equivalente a um coeficiente de correlação entre as distâncias observadas com as distâncias reproduzidas pela configuração final sendo esperado valores altos (Kruskal, 1964). 
Ricardo Primi, psicólogo, doutor em Psicologia Escolar e do Desenvolvimento Humano pela Universidade de São Paulo/Yale University (EUA), é professor do Curso de Graduação e do Programa de Estudos Pós-Graduados em Psicologia da Universidade São Francisco e Coordenador do Laboratório de Avaliação Psicológica e Educacional (LabAPE).

Acácia A. Angeli dos Santos, psicóloga, doutora em Psicologia Escolar e do Desenvolvimento Humano pela Universidade de São Paulo, é professora do Curso de Graduação e do Programa de Estudos Pós-Graduados em Psicologia da Universidade São Francisco.

Claudette Medeiros Vendramini, estatística, doutora em Educação pela Universidade Estadual de Campinas (UNICAMP), é professora do Curso de Graduação e do Programa de Estudos Pós-Graduados em Psicologia da Universidade São Francisco, São Paulo.

Endereço para correspondência: [RP] Universidade São Francisco, Laboratório de Avaliação Psicológica e Educacional, LabAPE, Mestrado em Psicologia, Rua Alexandre Rodrigues Barbosa, 45, 13251-900, Itatiba, SP. Tel. (11) 45348002. E-mail: [RP] rprimi@uol.com.br; [AAAS] acacia@sãofrancisco.edu.br; [CMV] vendramini@saofrancisco.edu.br. 